\title{
The STATISTICAL CoRrelation BETWEeN DISTORTION AND ADDING SIGNAL FOR PAPR REDUCTION IN OFDM BASED COMMUNICATION SYSTEMS
}

\author{
Désiré Guel ${ }^{1}$, Boureima Zerbo ${ }^{2}$, Jacques Palicot ${ }^{3}$ and Oumarou Sié ${ }^{1}$ \\ ${ }^{1}$ Department of Computer Engineering, \\ Joseph Ki-Zerbo University, Burkina Faso \\ ${ }^{2}$ Thomas-Sankara University, Burkina Faso \\ ${ }^{3}$ CentraleSupélec/IETR, Campus de Rennes, France
}

\begin{abstract}
In recent past years, PAPR (Peak-to-Average Power Ratio) of OFDM (Orthogonal FrequencyDivision Multiplexing) system has been intensively investigated. Published works mainly focus on how to reduce PAPR. Since high PAPR will lead to clipping of the signal when passed through a nonlinear amplifier. This paper proposes to extend the work related to "Gaussian Tone Reservation Clipping and Filtering for PAPR Mitigation" which has been previously published. So, in this paper, we deeply investigate the statistical correlation between PAPR reduction, and the distortion generated by three (3) adding signal techniques for PAPR reduction. Thereby, we first propose a generic function for PAPR reduction. Then, we analyse the PAPR reduction capabilities of each PAPR reduction technique versus the distortion generated. The signal-to-noise-and-distortion ratio (SNDR) metric is used to evaluate the distortion generated within each technique by assuming that OFDM baseband signals are modelled by complex Gaussian processes with Rayleigh envelope distribution for a large number of subcarriers. The results related to one of the techniques is proposed in the first time in this paper, unlike those related to the other two PAPR reduction techniques where the studies were already published. Comparisons of the proposed approximations of SNDR with those obtained by computer simulations show good agreement. An interesting result highlighted in this paper is the strong correlation existing between PAPR reduction performance and distortion signal power. Indeed, the results show that PAPR reduction gain increases as the distortion signal power increases. Through these 3 examples of PAPR reduction techniques; we could derive the following conclusion: in an adding signal context, the adding signal for PAPR reduction is closely linked to the distortion generated, and a trade-off between PAPR-reduction and distortion must be definitely found.
\end{abstract}

\section{KEYWORDS}

Orthogonal Frequency Division Multiplexing (OFDM), Peak-to-Average Power Ratio (PAPR), signal-to-noise-and-distortion ratio (SNDR), Adding Signal Techniques.

\section{INTRODUCTION}

Orthogonal Frequency Division Multiplexing (OFDM), a popular type of multicarrier transmission $[5,6,7,8]$, is an effective modulation technique for high-data-rate wireless and wireline applications, including Digital Subscriber Line (DSL) [9], Digital Audio Broadcasting (DAB) [10], Digital Video Broadcasting (DVB) [11], and Wireless Local Area Network David C. Wyld et al. (Eds): CSEA, DMDBS, NSEC, NETWORKS, Fuzzy, NATL, SIGEM - 2020 pp. 81-96, 2020. CS \& IT - CSCP 2020 
(WLAN) [12]. It has been adopted by the third-generation partnership program long-term evolution (3GPP-LTE) [13] and LTE-Advance. OFDM has been recently adopted for use in future 5G air interface [14]. One of the advantages of OFDM modulations is its ability to minimize the multi-path propagation effects and the impulse noise by transforming effectively the frequency selective fading channel into a flat fading channel. In addition, OFDM eliminates the need for equalizers and uses modern digital signals processing techniques, such as the fast Fourier transform (FFT) techniques [15].

Due to the large number of subcarriers, the OFDM signal has a large Peak-to-Average Power Ratio (PAPR) which makes system performance very sensitive to nonlinear distortions $[16,18]$. This remains one of the major drawbacks associated to OFDM transmission technique. In practice, transmission devices such as the High-Power Amplifier (HPA) have a limited dynamic range [19]. Therefore, to ensure a distortion-less transmission, hardware with a high-power backoff is required. But it restricts the OFDM system from utilizing the transmitted power effectively. To reduce the PAPR of OFDM signals, various techniques have been proposed [20]. The most widely known techniques are based on selective mapping [21], phase shifting [22] or some form of coding. Selective mapping and phase shifting offer a considerable reduction of the PAPR [21] but, at the price of a significant increase of the overall system complexity. Coding techniques with the capabilities of both PAPR reduction, as well as error correction (Reed-Muller codes) [23], are attractive. However, these codes significantly reduce the overall throughput of the system, especially if there is a relatively large number of subcarriers [23].

The novelty of this paper, on one hand, involves proposing a generic function for PAPR reduction which embeds three methods for PAPR reduction : SC [2], HC [3] and GM [4] that have been widely studied in the past; on the other hand, it involves investigating the relation between PAPR reduction and the distortion signal power by theoretical analysis and computer simulations which follows up the work done in [1]. So, the metrics such as complementary cumulative distribution function (CCDF) is used PAPR reduction performance evaluation. Signal-to-noise-and-distortion ratio (SNDR) metric is used to evaluate the distortion generated by the different schemes for PAPR reduction. What is also new is the proposed approximation of SNDR for GM [4] which is proposed the first time in this article, while those related to SC [2], HC [3] have been the studied and been published in [1]. Through these three (3) examples of PAPR reduction techniques; it has been demonstrated that adding signal for PAPR reduction is closely linked to the distortion generated, and a trade-off between PAPR-reduction and distortion must be found. Indeed, greater will be the PAPR reduction, greater will be the distortions generated that could affect the over-all system performance in term of BLER/BER degradation and OOB (Out-Off-Band) radiation/interference.

The paper is organized as follows. Section 2 introduces the related work. Section 3 characterizes the OFDM system and defines the PAPR in OFDM signals context. The three (3) adding signal techniques for PAPR reduction and distortion characterization are described in section 4 . In section 5, simulation results are shown, and conclusions are drawn in section 6. Some future directions and open problems are discussed in section 7.

\section{RELATED WORK}

In this section, we highlight works done in the past mainly related to "Geometric Method"(GM) which is strongly related to this paper; also, the Bussgang's principle are highlighted and will undoubtedly allow to understand the conclusions of our study. Due to the simplest way for PAPR reduction, the techniques in $[24,26,27,28]$ have been proposed and seem to be promising for use in commercial systems. In [28], it is an adding signal technique whose its adding signal for PAPR reduction is directly expressed; [24, 26, 27] are clipping techniques and considered, in this paper, 
as adding signal techniques whose the adding signal for PAPR reduction are the difference between original signal and its clipped version. All these techniques [24, 26, 27, 28] are nonlinear processes and may lead to significant distortion (in-band and out of band distortion) and performance loss. In [25], filtering is used to remove the out-of-band distortion whereas in [28], filtering is used to remove the part of adding signal generated over the OFDM signal bandwidth. This results in, no spectral regrowth in $[24,26]$ and no Bit Error Rate (BER) performance degradation in [28]. Recently, [29] presents a comparative analysis for various companding based PAPR reduction techniques and provides useful guidelines for the design and development of power and bandwidth efficient OFDM systems.

\subsection{The "Geometric Method"}

The "Geometric Method"(GM) has been proposed in [4] where the PAPR reduction scheme has been presented; the performance (PAPR reduction, transmission quality and execution complexity) has been presented as well in the context of a WLAN system based on the IEEE $802.11 \mathrm{a} / \mathrm{g}$ standard. The simulation results showed a loss of PAPR reduction performance when the PAPR reduction signal ("adding signal for PAPR") was set far from the OFDM signal in frequency domain. It has been demonstrated that the loss of PAPR reduction gain was due the distortion generated within the technique; so setting the PAPR reduction signal far from the OFDM signal in frequency domain in order to mitigate the generated distortion, at the same reduces PAPR reduction gain. However [4] did not highlight the relationship which existed between the PAPR reduction signal and the transmission quality degradation in term of BLER/BER.

In order to overcome the limitations of GM [4], an Enhanced GM (EGM) which is a downwardcompatible PAPR reduction technique has been proposed in [25]; the performance (reduction of PAPR, quality of transmission and complexity of execution) of the technique has been evaluated and compared to GM [4]. The results showed that, under the same simulation conditions, GM [4] is more efficient than EGM [25] in terms of PAPR reduction. However, EGM does not degrade BLER/BER unlike GM. The main reason of this outcome is that EGM filters the signal for PAPR reduction in order to preserve the BLER/BER; this results to in loss of PAPR reduction performance. EGM [25] remains a downward-compatible "adding signal" for PAPR reduction technique with none BLER/BER degradation. However, EGM does not control Out-Of-Band (OOB) spectral regrowth; this could lead to OOB (Out-Off-Band) radiation/interference. Therefore, in [25], an Enhanced "Geometric Method" based on "Tone Reservation" has been proposed. It transforms the GM [4] technique into the TR technique by filtering the PAPR reduction signal with an FFT/IFFT pair based digital filtering [37].

The main idea in [37] was to transform the adding signal techniques (like clipping techniques, GM) into TR techniques thanks to a filtering based on FTT/IFFT pair; the reason is to take benefit of the TR advantages such as none BLER/BER degradation, none Side Information (SI) transmission. As the transformation is a low-complexity process (about the FFT/IFFT complexity), the obtained technique results in a low-complexity TR technique. However, the transformation into TR techniques generates a loss of performance in PAPR reduction; it has been proposed to increase the PAPR reduction gain by iterating the process of transformation. However, the system complexity grows linearly with the number of iterations and a trade-off shall be done between the PAPR reduction performance and the PAPR reduction scheme's complexity. All these observations and PAPR reduction signal filtering seemed to indicate the link between the PAPR reduction signal and the distortion generated which affects BLER/BER performance and could lead to OOB radiation/interference. 


\subsection{Bussgang's principle}

In a report dating from March 1952 [31], J.J. Bussgang discusses the Gaussian signals (inter and auto) correlation function estimation, when one of the two inputs of the correlator undergo to a nonlinear transformation without memory. He succeeds in expressing the link between the intercorrelation function of these two signals and that of the correlation function before distortion. Bussgang's theorem has long been used and continues to be in the adaptive equalization area as evidenced by the works proposed in [32, 33]. In [32], Z. Ding and RA Kennedy deal with the role of the AM / AM transfer characteristic in non-linear systems with memory effect and propose an extension of the theorem of Bussgang in the case of complexes.

Now, assuming a nonlinear technique characterizes by its AM/AM nonlinear function $f($.$) ,$ where the output signal $y(t)$ is expressed as:

$$
\mathrm{y}(\mathrm{t})=f\left[r(t) e^{j \varphi(t)}\right], 0 \leq t \leq T_{s}
$$

The Bussgang's theorem [31] applied to equation (1) leads to:

$$
\mathrm{y}(\mathrm{t})=\alpha x(t)+d(t) \underset{\text { where, }}{\alpha=\frac{R_{y x}(0)}{R_{x x}(0)}}
$$

where the distortion term $d(t)$ is uncorrelated with the input signal $x(t)$. Using (2), the output correlation function of the signal $y(t)$ is expressed as:

$$
R_{y y}(\tau)=|\alpha|^{2} R_{x x}(\tau)+R_{d d}(\tau)
$$

The attenuation coefficient $\alpha$ is given by;

$$
\alpha=\frac{R_{y x}(0)}{R_{x x}(0)}=\frac{E\left[x^{*}(t) y(t)\right]}{P_{x}}=\frac{E[r f(r)]}{P_{x}}
$$

where $R_{y x}(\tau)$ denotes the input-output cross-correlation function, $f(r)$ the AM/AM non-linear function, $P_{x}$ is the OFDM signal power, and $E[\cdot]$ is the statistical expectation operator.

Different approaches may be used to compute $R_{y y}(\tau)$ including the Direct Method, the Transform Method, or the Derivative Method. In [17, 18], the Derivative Method is used, and it is possible to prove for any nonlinear function $f($.$) that:$

$$
R_{y y}(\tau)=\sum_{n=0}^{\infty} c_{n}\left[\frac{R_{x x}(\tau)}{P_{x}}\right]^{2 n+1}=\frac{c_{0}}{P_{x}} R_{x x}(\tau)+\sum_{n=1}^{\infty} c_{n}\left[\frac{R_{x x}(\tau)}{P_{x}}\right]^{2 n+1}
$$


with $c_{n}$ expressed by:

$$
c_{n}=\frac{1}{P_{x}} \frac{1}{n+1}\left\|\int_{D(r)} r f(r) p(r) \cdot L_{n}^{(I)}\left(\frac{r^{2}}{P_{x}}\right) d r\right\|^{2}
$$

where $D(r)=\{r: 0 \leq r \leq \infty\}$ is the domain of integration $p(r)$ is the probability density function of the OFDM envelope and $L_{n}^{(I)}(x)$ is the Laguerre function expressed by

$$
L_{n}^{(I)}(x)=\frac{x^{-k} e^{x}}{n !}\left(\frac{d}{d x}\right)^{n}\left(x^{n+k} e^{-x}\right)
$$

\section{OFDM SYSTEM AND PAPR}

In OFDM systems, the time-domain signal $x(t)$ can be written as

$$
x(t)=\frac{1}{\sqrt{N}} \sum_{k=0}^{N-1} X_{k} e^{2 j \pi \frac{k}{T_{s}} t}, 0 \leq t \leq T_{s}
$$

where $X_{k}$ is mapped data for $k=0,1,2, \ldots, N-1, N$ is the number of subcarriers and $T_{s}$ is the OFDM symbol period. The PAPR of $x(t)$ can be defined as

$$
\operatorname{PAPR}[x] \square \frac{\max _{t \in\left[0, T_{s}\right]}|x(t)|^{2}}{\mathrm{P}_{\mathrm{av}}}
$$

where $\mathrm{P}_{\mathrm{av}}$ is the average power defined as $\mathrm{P}_{\mathrm{av}}=\mathrm{E}\left[|x(t)|^{2}\right]$. Note that, it is more useful to consider $\operatorname{PAPR}[x]$ as a random variable and can be evaluated by using a statistical description given by the complementary cumulative density function (CCDF), defined as the probability that PAPR exceeds $\psi_{0}$, i.e., $\operatorname{CCDF}=\operatorname{Pr}\left\{\operatorname{PAPR}[x]>\psi_{0}\right\}$. To approximate PAPR in (9) in discrete time-domain, an oversampled version of (8) can be used. In this case, the oversampled timedomain signal $x_{n}$ can be written as

$$
x_{n}=\frac{1}{\sqrt{N}} \sum_{k=0}^{N-1} X_{k} e^{2 j \pi k \frac{n}{N L}}, 0 \leq n \leq N L-1
$$

where $L$ is the oversampling factor To better approximate the PAPR of continuous-time OFDM signals, $L \geq 4$ is used to capture the peaks of the continuous time-domain signals. The time- 
domain samples $x_{n}$ are NL-point IFFT of the data block with $(L-1) N$ zero-padding. The PAPR computed from the $L$-times oversampled time domain OFDM signal samples can be defined as:

$$
\operatorname{PAPR}[x] \square \frac{\max _{0 \leq n<N L}\left|x_{n}\right|^{2}}{\mathrm{E}\left[\left|x_{n}\right|^{2}\right]}
$$

\section{HC, SC AND GM ALgORITHMS AND DISTORTION CHARACTERIZATION}

Let us consider the discrete-time OFDM symbol $x_{n}$, the aim of adding signal techniques is to generate an adding signal $c_{n}$ such as $\operatorname{PAPR}\left[y_{n}\right]<\operatorname{PAPR}\left[x_{n}\right]$, where $y_{n}=x_{n}+c_{n}$ is the PAPR reduced signal. The adding signal $c_{n}$ for PAPR reduction is a function of the OFDM symbol $x_{n}$, i.e., $c_{n}=f\left(x_{n}\right)$ where $f($.$) is the adding signal generator function.$

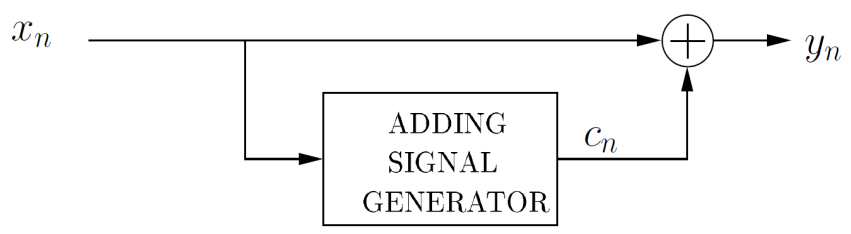

Figure 1: Adding signal scheme for PAPR reduction.

Fig.1 shows, the scheme of adding signal techniques for PAPR reduction.

\subsection{HC, SC and GM Characterization and SNDR Definition}

Now, let us consider the adding signal techniques, SC [2], HC [3] and GM [4] for PAPR reduction. The adding signal for PAPR reduction ${ }^{c_{n}}$ can be formulated as

$c_{n}=f_{\eta, \theta_{n}}\left(x_{n}\right)=\left\{\begin{array}{cc}(\eta-1) x_{n} e^{j \theta_{n}}, & \left|x_{n}\right|<\frac{1}{\eta} A \\ \left(\frac{A}{\left|x_{n}\right|}-1\right) x_{n} e^{j \theta_{n}}, & \left|x_{n}\right| \geq \frac{1}{\eta} A\end{array}\right.$,

where $A$ is the magnitude threshold, $\theta_{n}$ is defined as $\theta_{n}=2 \pi \delta f \frac{n}{N L} T_{s}, \quad 0 \leq n \leq N L-1$, and $T_{s}$ is the symbol period of the OFDM signal. The GM corresponds to (12) with $\eta=1$ and the parameter $\delta f$ is defined as $\delta f=f_{r}-f_{c}$, where $f_{r}$ and $f_{c}$ are the carrier frequencies of the OFDM signal and adding signal respectively; the SC is a special case of (12) with $\eta=1$ and $\delta f=0$; while the $\mathrm{HC}$ is a special case of (12) with $\eta=\infty$ and $\delta f=0$ 
These three (3) adding signal techniques for PAPR reduction, SC [2], HC [3] and GM [4] are all nonlinear processes and can be modelled as nonlinear AM/AM distorting functions $g_{\eta, \theta_{n}}($. defined below.

$$
g_{\eta, \theta_{n}}(x)=\left[1+(\eta-1) e^{j \theta_{n}}\right] x \cdot 1_{\substack{x<\frac{1}{\eta} A \\ \text { f }}}+\left[x+(1-x) e^{j \theta_{n}}\right] \cdot 1_{x \geq \frac{1}{\eta} A}
$$

It should be noted that similar threshold functions $g_{\eta, \theta_{n}}(x)$ have been also considered in other complex nonlinear dynamics [30]. Using (13), the PAPR reduced signal $y_{n}$ can be written as

$$
y_{n}=g_{\eta, \theta_{n}}\left(\left|x_{n}\right|\right) e^{j \varphi_{n}}=x_{n}+f_{\eta, \theta_{n}}\left(x_{n}\right)
$$

where $\varphi_{n}$ is the $x_{n}$ phase. According to Bussgang's theorem [31], the nonlinear functions $g_{\eta, \theta_{n}}($.$) can be decomposed as$

$$
g_{\eta, \theta_{n}}(x)=\alpha_{\eta, \theta_{n}} x+d
$$

where $d$ is the distortion created by $g_{\eta, \theta_{n}}($.$) and \alpha_{\eta, \theta_{n}}$ is chosen such that $d$ is uncorrelated with $x$, i.e., $\mathrm{E}\left[x d^{*}\right]=0$. From (15), it is shown that

$$
\alpha_{\eta, \theta_{n}}=\frac{\mathrm{E}\left[x^{*} g_{\eta, \theta_{n}}(x)\right]}{E\left[|x|^{2}\right]}=\frac{\mathrm{E}\left[x^{*} g_{\eta, \theta_{n}}(x)\right]}{\mathrm{P}_{\mathrm{av}}}
$$

The distortion power is given by

$$
\varepsilon_{d}=\mathrm{E}\left[|d|^{2}\right]=\mathrm{E}\left[\left|g_{\eta, \theta_{n}}(x)\right|^{2}\right]-\left|\alpha_{\eta, \theta_{n}}\right|^{2} \mathrm{P}_{\mathrm{av}} .
$$

The nonlinear functions $g_{\eta, \theta_{n}}($.$) depend on the samples \theta_{n}$, thus we define $\operatorname{SNDR}_{\left[\theta_{n}\right]}$ as the signal-to-noise-and-distortion ratio for $\theta_{n}$, given.

$$
\operatorname{SNDR}_{\left[\theta_{n}\right]}=\frac{\left|\alpha_{\eta, \theta_{n}}\right|^{2} \mathrm{P}_{\mathrm{av}}}{\mathrm{N}_{0}+\varepsilon_{d}}=\operatorname{SNR} \frac{\left|\alpha_{\eta, \theta_{n}}\right|^{2}}{1+\operatorname{SNR} \frac{\varepsilon_{d}}{\mathrm{P}_{\mathrm{av}}}}
$$

where $\mathrm{SNR}=\mathrm{P}_{\mathrm{av}} / \mathrm{N}_{0}$ is the signal-to-noise ratio (SNR).The global SNDR is thus defined as 


$$
\mathrm{SNDR}=\frac{1}{N} \sum_{\theta_{n}} \operatorname{SNDR}_{\left[\theta_{n}\right]}
$$

\subsection{SNDR Close Form of the Three Adding Signal Techniques}

Assuming that the baseband OFDM signal converges to a complex Gaussian process for large N, it has been shown in [34] that $\left|x_{n}\right|$ is an i.i.d Rayleigh random variable of which the probability density function (PDF) is :

$$
p_{X}(x)=\frac{2 x}{\mathrm{P}_{\mathrm{av}}} e^{-\frac{x^{2}}{\mathrm{P}_{\mathrm{av}}}}, \quad x \geq 0
$$

To derive a SNDR close form of the three adding signal techniques for PAPR reduction, we consider the equations (15) to (19) where the variable $x$ is an i.i.d Rayleigh random variable whose PDF has been given in (20).

Let $\mathrm{M}_{\eta}$ and $\mathrm{N}_{\eta}$ the expressions defined as

$$
\mathbf{M}_{\eta}=\int_{0}^{\frac{1}{\eta} A} x^{2} p_{X}(x) d x \quad \mathrm{~N}_{\eta}=\frac{A}{\eta} \int_{\frac{1}{\eta} A}^{\infty} x p_{X}(x) d x
$$

We show by developing (21) and using (20) that

$$
\mathrm{M}_{\eta}=\left[1-\left(1+\frac{A^{2}}{\eta^{2} \mathrm{P}_{\mathrm{av}}}\right) e^{-\frac{A^{2}}{\eta^{2} \mathrm{a}_{\mathrm{av}}}}\right] \mathrm{P}_{\mathrm{av}}, \quad \mathrm{N}_{\eta}=\left[\frac{A^{2}}{\eta^{2} \mathrm{P}_{\mathrm{av}}} e^{-\frac{A^{2}}{\eta^{2} \mathrm{P}_{\mathrm{av}}}}+\frac{A \sqrt{\pi}}{\eta \sqrt{\mathrm{P}_{\mathrm{av}}}} Q\left(\frac{A \sqrt{2}}{\eta \sqrt{\mathrm{P}_{\mathrm{av}}}}\right)\right] \mathrm{P}_{\mathrm{av}}
$$

where $Q($.$) is the Q-function defined as Q(x)=\frac{1}{\sqrt{2 \pi}} \int_{x}^{\infty} e^{-\frac{\tau^{2}}{2}} d \tau$

Now, using the definition of $\mathrm{M}_{\eta}$ and $\mathrm{N}_{\eta}$ given in (21), we can very easily show that

$$
\mathrm{E}\left[x^{*} g_{\eta, \theta_{n}}(x)\right]=\int_{0}^{\infty} x^{*} g_{\eta, \theta_{n}}(x) p_{X}(x) d x=\left(1-e^{j \theta_{n}}\right) \mathrm{P}_{\mathrm{av}}+\eta\left(\mathrm{M}_{\eta}+\mathrm{N}_{\eta}\right) e^{j \theta_{n}},
$$

and 


$$
\begin{aligned}
\mathrm{E}\left[\left|g_{\eta, \theta_{n}}(x)\right|^{2}\right] & =\int_{x}^{\infty}\left|g_{\eta, \theta_{n}}(x)\right|^{2} p_{X}(x) d x \\
& =\left|1-(\eta-1) e^{j \theta_{n}}\right|^{2} \mathrm{M}_{\eta}+\frac{A^{2}}{\mathrm{P}_{\mathrm{av}}} e^{-\frac{A^{2}}{\eta^{2} \mathrm{P}_{\mathrm{av}}}} \mathrm{P}_{\mathrm{av}}+2\left(1-\cos \theta_{n}\right)\left[\mathrm{P}_{\mathrm{av}}-\left(\mathrm{M}_{\eta}+\eta \mathrm{N}_{\eta}\right)\right]
\end{aligned}
$$

Now, substituting (22) into (23) and (24), we show that

$$
\left|\alpha_{\eta, \theta_{n}}\right|^{2}=1+\left[1-\eta\left(1-\Gamma\left(\frac{A}{\eta \sqrt{\mathrm{P}_{\mathrm{av}}}}\right)\right)\right]^{2}-2\left[1-\eta\left(1-\Gamma\left(\frac{A}{\eta \sqrt{\mathrm{P}_{\mathrm{av}}}}\right)\right)\right] \cos \theta_{n}
$$

And

$$
\frac{\varepsilon_{d}}{\mathrm{P}_{\mathrm{av}}}=\eta^{2}\left(1-e^{-\frac{A^{2}}{\eta^{2} \mathrm{P}_{\mathrm{av}}}}\right)-\eta^{2}\left(1-\Gamma\left(\frac{A}{\eta \sqrt{\mathrm{P}_{\mathrm{av}}}}\right)\right)^{2},
$$

Where $\Gamma(x)$ is the function defined as $\Gamma(x)=e^{-x^{2}}-x \sqrt{\pi} Q(x \sqrt{2}), \quad x \geq 0$.

\subsubsection{Case of Soft-Clipping (SC) [2]}

Setting $\eta=1$ and $\delta f=0$ (i.e., $\theta_{n}=0$ ) in (25) and (26); and substituting (25) and (26) into (18), we obtain the SNDR for SC expressed by (27).

$$
\mathrm{SNDR}=\mathrm{SNR} \frac{\left(1-\Gamma\left(\frac{A}{\sqrt{\mathrm{P}_{\mathrm{av}}}}\right)\right)^{2}}{1+\mathrm{SNR}\left(1-e^{-\frac{A^{2}}{\mathrm{P}_{\mathrm{av}}}}-\left(1-\Gamma\left(\frac{A}{\sqrt{\mathrm{P}_{\mathrm{av}}}}\right)\right)^{2}\right)},
$$

\subsubsection{Case of Heavyside-Clipping (HC) [3]}

When $\eta=\infty$ and $\delta f=0$, we have the HC case. Let us start by setting $\delta f=0$ (i.e., $\theta_{n}=0$ ) in (25) ; we obtain

$\left|\alpha_{\eta, \theta_{n}}\right|^{2}=\eta^{2}\left(1-\Gamma\left(\frac{A}{\eta \sqrt{\mathrm{P}_{\mathrm{av}}}}\right)\right)^{2}$

Due to $\eta\left(1-\Gamma\left(\frac{A}{\eta \sqrt{\mathrm{P}_{\mathrm{av}}}}\right)\right) \square \frac{A}{2} \sqrt{\frac{\pi}{\mathrm{P}_{\mathrm{av}}}}$ and $\eta \sqrt{1-e^{-\frac{A^{2}}{\eta^{2} \mathrm{P}_{\mathrm{av}}}}} \sqcap \frac{A}{\sqrt{\mathrm{P}_{\mathrm{av}}}}$ for $\eta \square \infty$, the SNDR for HC is given by 


$$
\mathrm{SNDR}=\mathrm{SNR} \frac{\frac{\pi}{4} \frac{A^{2}}{\mathrm{P}_{\mathrm{av}}}}{1+\mathrm{SNR}\left(\left(1-\frac{\pi}{4}\right) \frac{A^{2}}{\mathrm{P}_{\mathrm{av}}}\right)},
$$

\subsubsection{Case of Geometric Method (GM) [4]}

The GM corresponds to (12) with $\eta=1$ and the parameter $\delta f$ is defined as $\delta f=f_{r}-f_{c}$. Setting only $\eta=1$ in (25) and (26); we obtain

$$
\mathrm{SNDR}_{\left[\theta_{n}\right]}=\mathrm{SNR} \frac{1-2 \Gamma\left(\frac{A}{\sqrt{\mathrm{P}_{\mathrm{av}}}}\right) \cos \theta_{n}-\Gamma^{2}\left(\frac{A}{\sqrt{\mathrm{P}_{\mathrm{av}}}}\right)}{1+\mathrm{SNR}\left(1-e^{-\frac{A^{2}}{\mathrm{P}_{\mathrm{av}}}}-\left(1-\Gamma\left(\frac{A}{\sqrt{\mathrm{P}_{\mathrm{av}}}}\right)\right)^{2}\right)},
$$

According to the sum of Riemann theorem, for large $N$

$$
\frac{1}{N} \sum_{n=0}^{N-1} \cos \theta_{n} \square \frac{1}{2 \pi \delta f T_{s}} \int_{0}^{2 \pi \delta f T_{s}} \cos \theta d \theta=\frac{\sin \left(2 \pi \delta f T_{s}\right)}{2 \pi \delta f T_{s}},
$$

Using (30) in (19), the SNDR for GM is derived by

$$
\mathrm{SNDR}, \mathrm{SNR} \frac{1-2 \Gamma\left(\frac{A}{\sqrt{\mathrm{P}_{\mathrm{av}}}}\right) \frac{\sin \left(2 \pi \delta f T_{s}\right)}{2 \pi \delta f T_{s}}-\Gamma^{2}\left(\frac{A}{\sqrt{\mathrm{P}_{\mathrm{av}}}}\right)}{1+\mathrm{SNR}\left(1-e^{-\frac{A^{2}}{\mathrm{P}_{\mathrm{av}}}}-\left(1-\Gamma\left(\frac{A}{\sqrt{\mathrm{P}_{\mathrm{av}}}}\right)\right)^{2}\right)}
$$

\section{Simulation ReSUlts}

Computer simulations were performed on an OFDM system with 64-subcarriers and 16- QAM symbol mapping; $10^{4}$ OFDM symbols were randomly generated. GM is applied to the OFDM system with $\delta f T_{s}=1 / 8$. Indeed, in [4], it is shown that, when $\delta f T_{s}$ approaches 1/8, GM realizes a significant PAPR reduction gain.

Fig. 2 compares the theoretical SNDR as a function of SNR with the simulation results for the three adding signal techniques. The proposed approximations diverge from the simulation results for a low SNR. However, for an SNR $\geq 5 \mathrm{~dB}$, they show good agreement with the simulation results. This is because as for a low SNR, the approximation error in (27), (28) and (31) becomes dominant. Let us note that, the accuracy of approximation can be improved by increasing the sampling rate. 


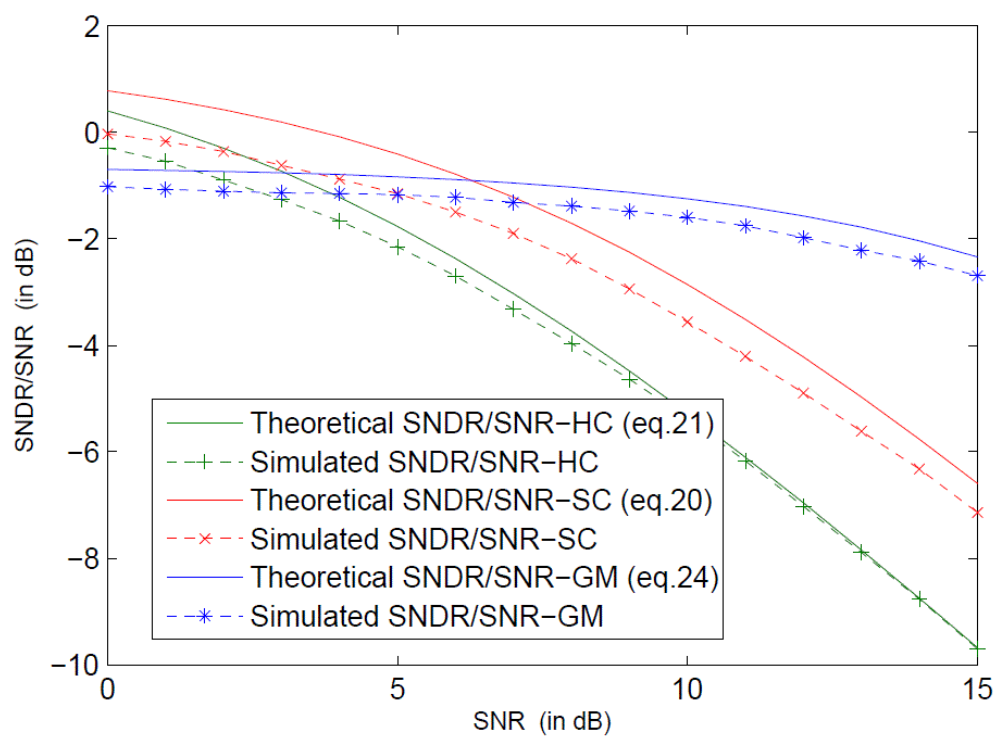

Figure 2: Comparison of the proposed SNDR as a function of SNR with the simulation results for $\frac{A}{\sqrt{\mathrm{P}_{\mathrm{av}}}}=3 \mathrm{~dB}$

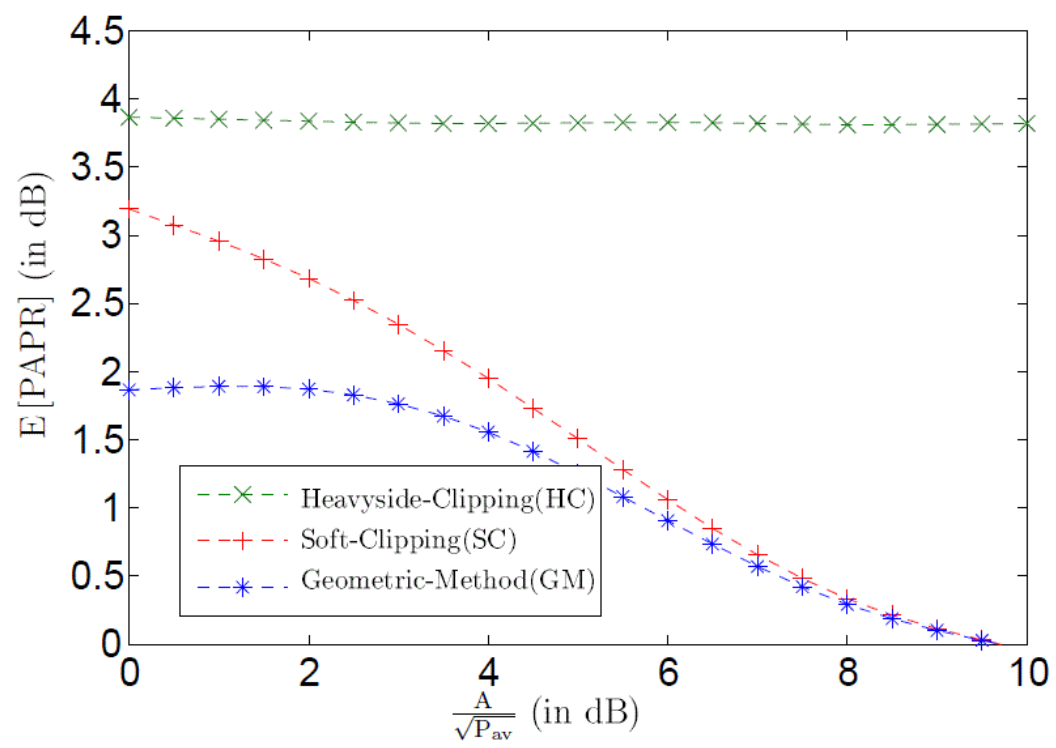

Figure 3: PAPR reduction performance as a function of $/ \sqrt{\mathrm{P}_{\mathrm{av}}}$ for $\mathrm{SC}, \mathrm{HC}$ and GM

Fig.3 plots the PAPR reduction performance of the three adding signal methods for PAPR reduction. In order to evaluate the PAPR reduction performance, E[ $\triangle \mathrm{PAPR}]$ defined as the difference between the initial PAPR and the reduced PAPR is used. For SC and GM, E[ $\triangle \mathrm{PAPR}]$ decreases as $/ \sqrt{\mathrm{P}_{\mathrm{av}}}$ increases, whereas for $\mathrm{HC}, \mathrm{E}[\triangle \mathrm{PAPR}]$ is almost constant. 


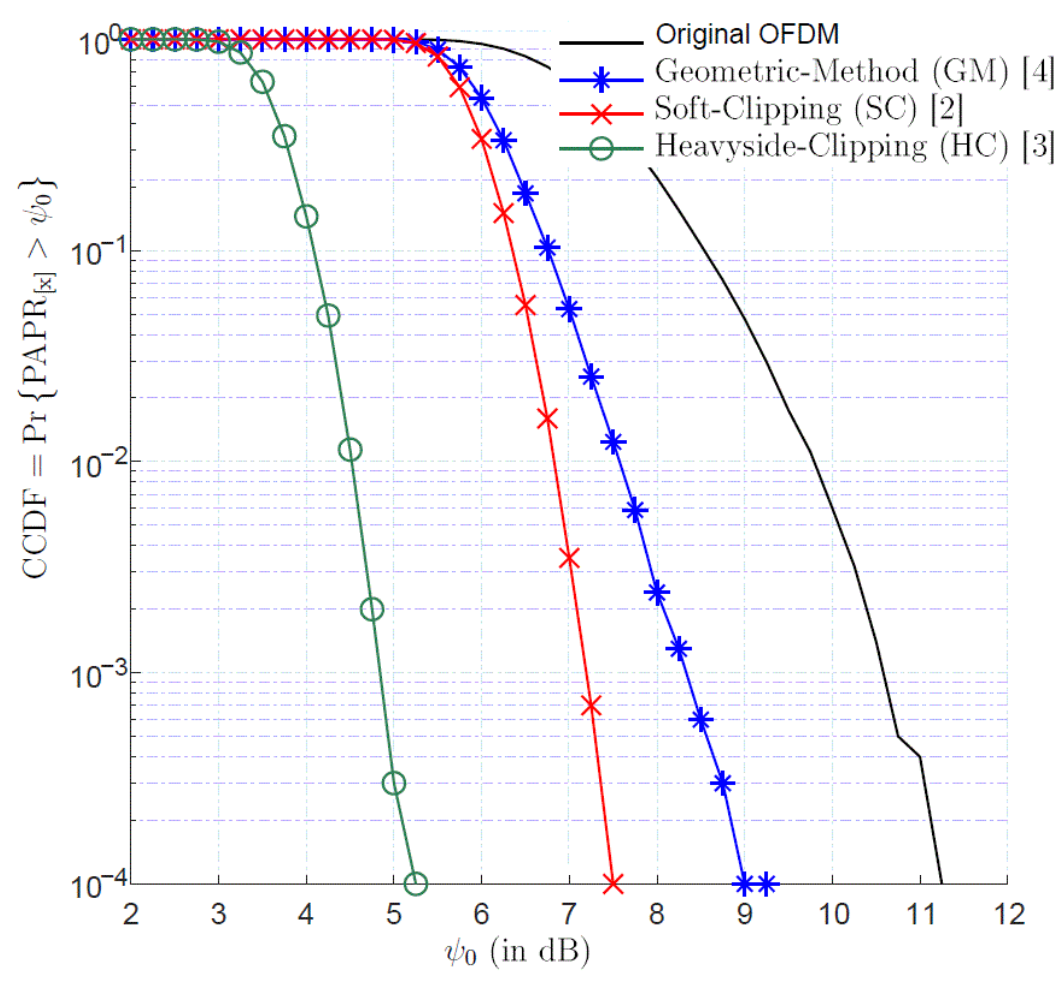

Figure 4: CCDF of SC, HC and GM for $/ \sqrt{\mathrm{P}_{\mathrm{av}}}=5 \mathrm{~dB}$.

Note that, for the same value of $/ \sqrt{\mathrm{P}_{\mathrm{av}}}, \mathrm{HC}$ is more efficient than $\mathrm{SC}$ which is more efficient than GM in terms of PAPR reduction as shown in Fig.4. For example in Fig.4, at $10^{-2}$ of the CCDF, HC reduces about $5.25 \mathrm{~dB}$ the PAPR, SC reduces about $2.75 \mathrm{~dB}$, whereas in GM case, the reduction is about $2.25 \mathrm{~dB}$.

Now, let us look at closely the simulation results about the distortion analysis. Fig.5 plots the simulated SNDR to SNR as a function of $/ \sqrt{\mathrm{P}_{\text {av }}}$ for the three different adding signal methods with SNR $=10 \mathrm{~dB}$. It shows that, for the same value of $/ \sqrt{\mathrm{P}_{\mathrm{av}}}, \mathrm{HC}$ introduces more distortion than SC which introduces more distortion than GM. For example, for $/ \sqrt{\mathrm{P}_{\mathrm{av}}}=3 \mathrm{~dB}$, SNDR/SNR is equal to $-5.5 \mathrm{~dB},-2.75 \mathrm{~dB}$ and $-2.25 \mathrm{~dB}$ for $\mathrm{HC}, \mathrm{SC}$ and $\mathrm{GM}$ respectively. 


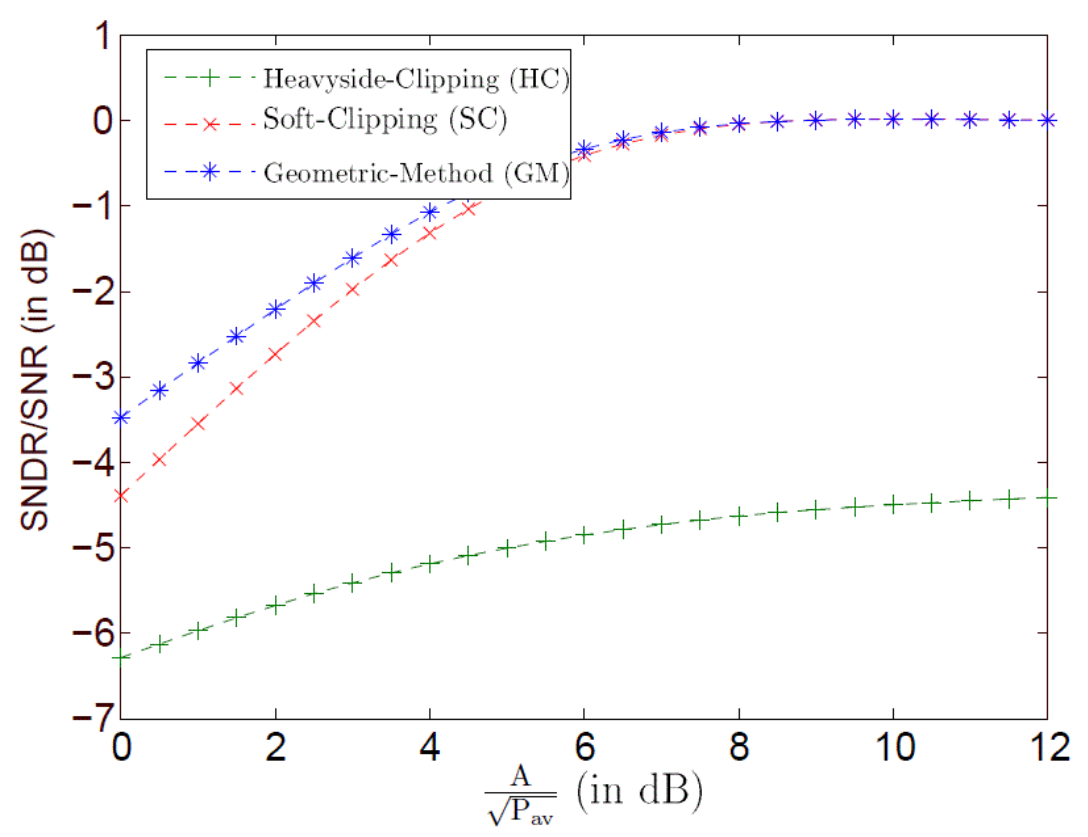

Figure 5: SNDR to SNR of SC, HC and GM for SNR $=10 \mathrm{~dB}$.

According to the results of Fig.3 and Fig.5, we remark that

- for SC and GM, larger is $A / \sqrt{\mathrm{P}_{\text {av }}}$, more significant will be the PAPR reduction and more significant will be the distortion generated,

- HC reduces greatly the PAPR but leads to a significant distortion generation.

With above remarks, we can conclude that, a significant distortion lead to a considerable reduction of the PAPR.

\section{Conclusion}

In this paper, we have proposed a generic function for PAPR reduction which embeds three (3) methods for PAPR reduction widely studied in the past : these three (3) methods are known also as adding signal techniques. The PAPR reduction performance of these three (3) adding signal techniques and the distortion generated by these techniques were studied. We analysed the distortion generated by deriving a close form expression of the SNDR while assuming that OFDM baseband signals are modelled by complex Gaussian processes with Rayleigh envelope distribution for a large number of subcarriers. The proposed approximation of SNDR for these adding signal techniques show good agreement with the simulation results. The results related to "Geometric Method" (GM) are proposed in the first time in this paper, unlike those related to Soft-Clipping (HC), Heavyside-Clipping (HC) where the studies were already published.

Comparisons between these techniques are made in terms of PAPR reduction performance and distortion generated. Results show that, the PAPR reduction increases as the distortion generated increases (in terms of power). 
From this study, we have drawn the following conclusion namely, in adding signal context, the adding signal for PAPR reduction is closely linked to the distortion generated. So, a trade-off between PAPR-reduction and distortion must be done.

\section{FUTURE WORK}

As it has been shown in this article through these 3 techniques, SC [2], HC [3] and GM [4], OFDM PAPR can be reduced at the price of more or less significant distortion generated which could affect BLER/BER performance and lead to OOB (Out-Off-Band) radiation/interference.

To follow up this work, the following directions have been identified and deserve to be developed:

- An optimal solution should be investigated in such way the PAPR will be significantly reduced at the price of "weak distortion" or "none distortion" generated. A single iteration should be considered in order to keep the PAPR reduction scheme low power consumption. As the PAPR reduction techniques investigated here are characterized by a nonlinear function $f_{\eta, \theta_{n}}($.$) which acts on the amplitude of the signals for PAPR reduction. It would$ be interesting to find the optimal PAPR reduction function $f_{\eta, \theta_{n}}^{o p t}($.$) which will lead to$ significant PAPR reduction with low computational complexity.

- A further direction for the work improvement is to combine the PAPR reduction techniques investigated in this paper with High Power Amplifier (HPA) linearization techniques especially with an adaptive predistortion method taking into account the memory effect of the amplifier. The goal should be to provide a big picture in terms of the overall system performance enhancement and not focus only on PAPR reduction techniques.

\section{ACKNOWLEDGMENT}

The authors would like to thank the authors and contributors whose works were included in this study.

\section{REFERENCES}

[1] Y. Louet, J. Palicot and D. Guel, "Gaussian Tone Reservation Clipping and Filtering for PAPR Mitigation", International Journal on Advances in Telecommunications, vol 12 no 1 \& 2, year 2019.

[2] X. Li and L.J. Cimini, "Effects of Clipping and Filtering on the Performance of OFDM," IEEE Communication Letters, vol. 2, no. 5, pp. 131-133, May 1998.

[3] Qian Hua, R. Raich, G.T. Zhou, "On the benefits of deliberately introduced baseband nonlinearities in communication systems", IEEE International Conference on Acoustics, Speech, and Signal Processing (ICASSP '04), vol. 2, pp. 905-908, May 2004.

[4] D. Guel, J. Palicot and Y. Louet, "A Geometric Method for PAPR Reduction in a Signal Adding Context for OFDM Signals", IEEE International Conference on Digital Signal Processing, pp. 347350, 1-4 July 2007.

[5] J. Bingham, "Multicarrier modulation for data transmission: an idea whose time has come,"IEEE Commun. Mag., vol. 28, pp. 5-14, May 1990.

[6] L. Litwin, “An introduction to multicarrier modulation,’IEEE Potentials, vol. 19, pp. 36-38, Apr.May 2000.

[7] Z.Wang and G. B. Giannakis, "Wireless multicarrier communications,"IEEE Signal Processing Mag., pp. 29-48, May 2000. 
[8] R. van Nee and R. Prasad, "OFDM Wireless Multimedia Communications.” London, UK: Artech House, 2000.

[9] “Asymmetrical Digital Subscriber Line (ADSL) Transceivers,” ITU-T Recommendation G.992.1, July 1999.

[10] ETSI, "Radio broadcasting systems: digital audio broadcasting to mobile, portable and fixed receivers", European Telecommunication Standard, ETS 300-401, Feb. 1995.

[11] ETSI, "Digital video broadcasting: framing structure, channel coding and modulation for digital terrestrial television", European Telecommunication Standard, ETS 300-744, Aug. 1997.

[12] IEEE 802.11, "IEEE standards for wireless LAN medium access control (MAC) and physical layer (PHY) specifications", Nov. 1997

[13] I. Pospishny, V. Vasyuk, S. Romanchyk, O. Dovzhenko, O. Dony and V. Shvaichenko, "3GPP long term evolution (LTE)," 2010 International Conference on Modern Problems of Radio Engineering, Telecommunications and Computer Science (TCSET), Lviv- Slavske, 2010, pp. 192-192.

[14] A. Ghosh, A. Maeder, M. Baker and D. Chandramouli, "5G Evolution: A View on 5G Cellular Technology Beyond 3GPP Release 15,'in IEEE Access, vol. 7, pp. 127639- 127651, 2019, doi: 10.1109/ACCESS.2019.2939938.

[15] S. B. Weinstein and P. M. Ebert, "Data transmission by frequency-division multiplex- ing using the discrete Fourier transform,'IEEE Trans. Commun. Technol., vol. 19, pp. 628-634, Oct. 1971.

[16] S. Merchan, A. G. Armada, and J. L. Garcia, “OFDM performance in amplifier non- linearity,”IEEE Trans. Broadcast., vol. 44, pp. 106-114, Mar. 1998.

[17] W.B. Davenport, W.L. Root, "An Introduction to the Theory of Random Signals and Noise", McGraw-Hill, 1958

[18] P. Banelli, G. Baruffa, and S. Cacopardi, "Effects of HPA nonlinearity on frequency multiplexed OFDM signals,'IEEE Trans. Broadcast., vol. 47, pp. 123-136, June 2001.

[19] A. Barbieri, G. Cariolaro, and L. Vangelista, "Nonlinear models of TWT revisited for OFDM systems," in Proc. of the 38 th Midwest Symposium on Circuits and Systems, vol. 1, Aug. 1996, pp. 522-525.

[20] Yves Louet and Jacques Palicot, "A classification of methods for efficient power amplification of signals", Annals of Telecom, vol. 63, nb. 7-8, pp. 351-368, Aug. 2008

[21] R. W. Buml, R. F. H. Fischer, and J. B. Huber, "Reducing the peak-to average power ratio of multicarrier modulation by selected mapping,"Electronics Letters, vol. 32, pp. 2056-2057, Oct. 1996.

[22] L. J. Cimini, Jr. and N. R. Sollenberger, "Peak-to-average power ratio reduction of an OFDM signal using partial transmit sequences,'IEEE Commun. Lett., vol. 4, pp. 86-88, Mar. 2000.

[23] J. Davis and J. Jedwab, "Peak-to-mean power control in OFDM, Golay complementary sequences, and Reed-Muller codes,'IEEE Trans. Inform. Theory, vol. 45, pp. 2397- 2417, Nov. 1999.

[24] J. Armstrong, "New OFDM peak-to-average power reduction scheme", Vehicular Technology Conference, VTC 2001 Spring. IEEE VTS 53rd, vol.1, 2001 pp. 756 - 760, 2001.

[25] D. Guel, J. Palicot, and Y. Louet, "Tone reservation technique based on geometric method for orthogonal frequency division multiplexing peak-to-average power ratio re- duction", IET Commun., November 2010 Volume 4, Issue 17, pp. 2065-2073.

[26] J. Armstrong, "Peak-to-average power reduction for OFDM by repeated clipping and frequency domain filtering," Electronics Letters, vol. 38, pp. 246-247, Feb. 2002.

[27] H. Ochiai and H. Imai, "On clipping for peak power reduction of OFDM signals, in IEEE Global Telecommun. Conf., 2000. (GLOBECOM'00), vol. 2, Nov. 27-Dec. 1, 2000, pp. 731-735.

[29] A. Mohammed, M. Shehata, A. Nassar and H. Mostafa, "Performance Comparison of CompandingBased PAPR Suppression Techniques in OFDM Systems," 8th International Conference on Modern Circuits and Systems Technologies (MOCAST) Year: 2019

[30] Yilun Shang, "Deffuant model of opinion formation in one-dimensional multiplex networks,"Journal of Physics A: Mathematical and Theoretical, 48(39), 395101 - October 2015, https://doi.org/10.1088/1751-8113/48/39/395101

[31] J. Bussgang, "Crosscorrelation function of amplitude-distorted Gaussian signals,"'Research laboratory of electronics, Massachusetts Institute of Technology, Cambridge. Technical Report 216, (1952).

[32] J. Minkoff, "The Role of AM-to-PM Conversion in Memoryless Nonlinear Systems," vol. 33, pp. 139-144, Feb 1985.

[33] J. B. D. Filho, G. Favier, and J. M. T. Romano, "New Bussgang methods for blind equalization," in Proc. IEEE International Conference on Acoustics, Speech, and Signal Processing ICASSP-97, vol. 3, pp. 2269-2272, 21-24 April 1997. 
[34] S. Wei, D. L. Goeckel, and P. A. Kelly, "The complex enveloppe of a bandlimited OFDM signal converges weakly to a Gaussian random process: proof and application," to appear in IEEE Transactions on Information Theory, http://www.ece.lsu.edu/swei.

[35] D.Guel and J. Palicot, "Clipping Formulated As an Adding Signal Technique for OFDM Peak Power Reduction,” VTC Spring 2009 - IEEE 69th Vehicular Technology Conference

[36] T. Jiang and Y. Wu, "An overview: Peak-to-average power ratio reduction techniques for OFDM signals,"IEEE Transactions on broadcasting, 54(2):257-268, 2008.

[37] D. Guel and J. Palicot, "FFT-IFFT Pair Based Digital Filtering for the Transformation of Adding Signal PAPR Reduction Techniques in Tone Reservation Techniques,"in International Conference on Wireless and Mobile Communications (ICWMC), 2009.

(C) 2020 By AIRCC Publishing Corporation. This article is published under the Creative Commons Attribution (CC BY) license. 